\title{
Life-threatening Hemoperitoneum Following Cardiac Arrest by Myocardial Infarction: A Case Report of Successful Endovascular Management
}

\section{José João Martins*, Laura Costa, Anabela Mesquita and Luís Lencastre}

Unidade de Cuidados Intensivos, Hospital de Braga, Braga, Portugal

*Corresponding Author: José João Martins, Unidade de Cuidados Intensivos, Hospital de Braga, Braga, Portugal.
Received: March 25, 2021

Published: May 18, 2021

(C) All rights are reserved by José João Martins., et al.

\begin{abstract}
Spontaneous hepatic artery rupture with hemoperitoneum and haemorrhagic shock is a life-threatening condition, the seriousness of this condition is multiplied if it follows another life-threatening event, in this case, a myocardial infarction with cardiac arrest. The following clinical case depicts a patient admitted to the intensive care unit following cardiac arrest, shortly after admission the patient becomes unstable with haemorrhagic shock that was found to be due a bleeding hepatic artery. The hemoperitoneum was controlled using atypical means with an endovascular approach. Our clinical case will hopefully demonstrate an atypical scenario where the source of clinical deterioration was not apparent and represents a very rare complication of cardiac resuscitation. This case demonstrates the need for a clinician to always be alert for rare complications and be able to quickly adapt.
\end{abstract}

Keywords: Hemoperitoneum; Cardiac Arrest; Endovascular Procedures; Hepatic Artery; Critical Care

\section{Introduction}

Spontaneous hepatic artery rupture with hemoperitoneum and haemorrhagic shock is a life-threatening condition, the diagnosis is especially difficult if the patient was admitted into the intensive care unit (ICU) with an unrelated diagnosis and is sedated and unable to communicate abdominal pain. The authors will present a clinical case of an hemoperitoneum due to hepatic artery rupture on a patient admitted for cardiac arrest due to myocardial infarction.

\section{Case Report}

The following clinical case depicts a healthy male of 58 years, with no known medical conditions that was victim of sudden cardiac arrest while sitting in a coffee shop. Due to the proximity of a medical team, cardiopulmonary resuscitation (CPR) was quickly initiated with the aid of an external defibrillator. A total of 8 minutes of CPR and 4 shocks were administered until spontaneous circulation was obtained. Initial evaluation on the spot by a pre- hospital emergency team that documented a myocardial infarction with ST-segment elevation of the inferior wall (Image 1). Following intubation for airway protection and initiation of mechanical ventilation, the patient was transported to the nearest hospital capable of percutaneous coronary intervention. Initial coronary angiography revealed a distal occlusion of the right coronary artery that was treated with angioplasty and placement of Sirolimus coated stent alongside initiation of double antiplatelet therapy and due to the presence of residual thrombosis distal to the stent location low molecular weight heparin was also initiated twice daily. The patient was admitted to the ICU for post-reanimation care and initiation of hypothermia.

On admission, the patient was sedated with propofol at 200 $\mathrm{mg} / \mathrm{h}$ and fentanyl at $50 \mathrm{mcg} / \mathrm{h}$ with a Richmond Agitation-Sedation scale of -5 , mechanically ventilated with a pressure regulated volume-controlled mode with normal $\mathrm{PaCO}_{2}$ and a normal $\mathrm{PaO}_{2} /$ $\mathrm{FiO}_{2}$ ratio. The patient was stable with a normal heart rate and necessitating noradrenaline at $0,12 \mathrm{mcg} / \mathrm{kg} / \mathrm{min}$ to maintain mean 
blood pressure above $65 \mathrm{mmHg}$. His urine output was around $1 \mathrm{ml} /$ $\mathrm{kg} / \mathrm{h}$ and his haemoglobin concentration on admission was $15,2 \mathrm{~g} /$ dL.

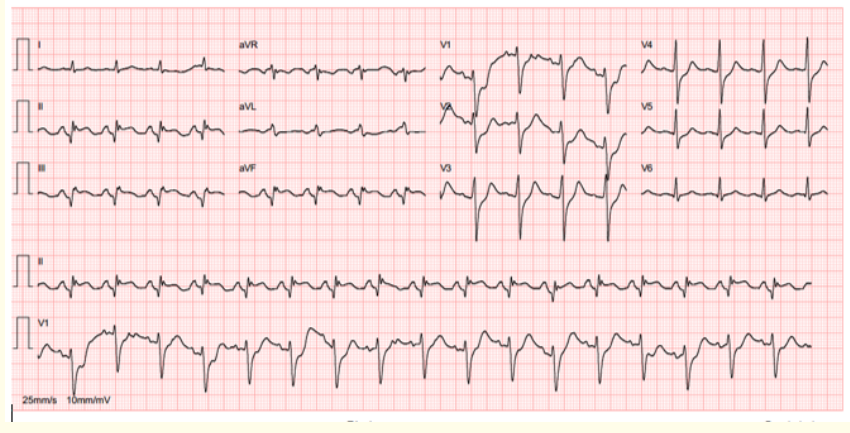

Image 1: Electrocardiogram showing ST-Segment elevation on the inferior wall derivations.

During the first twelve hours after admission, the patient developed hypotension, tachycardia and low urine output. A laboratory analyses revealed a fall of $5 \mathrm{~g} / \mathrm{dL}$ in the haemoglobin and norepinephrine was progressively increased until $1 \mathrm{mcg} / \mathrm{kg} / \mathrm{min}$. Clinical examination revealed a non-compressible abdomen and signs of low perfusion of peripheral tissues.

With a possible haemorrhagic shock due to active intra-abdominal bleeding, blood transfusion was initiated and the patient was quickly transported to the imaging department to perform a computed tomographic angiography of the abdomen and pelvis.

The exam revealed a large hemoperitoneum along the falciform ligament with imagological signs of active bleeding of the right hepatic artery (Image 2). Upon discussion with the surgical, radiology and ICU team a decision was reached to attempt treatment with an endovascular approach. The initial angiography showed active bleeding of the hepatic artery without a clear point of rupture. An extensive embolization with placement of coils was done on the proximal end of the left and right hepatic arteries and also along the common hepatic artery (Image 3). Once bleeding was controlled there was a marked improvement in the patient condition with an abrupt decrease in the need for vasopressors and an overall improvement of cardiovascular and renal function. In total 6 units of transfused blood were administered (around 1.2 Liters).

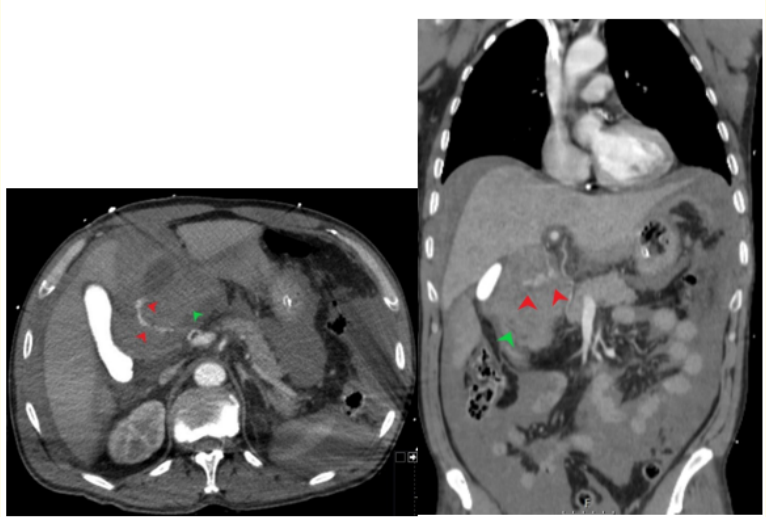

Image 2: Computed Tomography of the abdomen showing a diffuse hemoperitoneum with suspected origin in the hepatic artery.

Red arrows showing right hepatic artery with signs of active bleeding. Green arrow showing extensive hemoperitoneum.

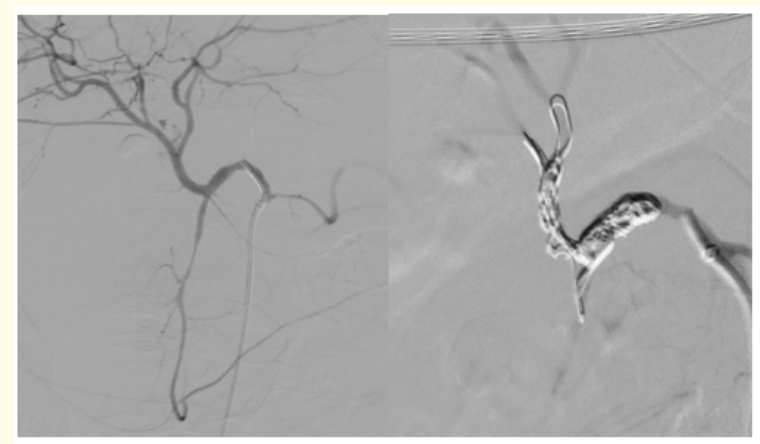

Image 3: Angiography images showing on the left both right and left hepatic arteries and the gastroduodenal artery without clear signs of rupture and on the right the angiography after coil placement.

The following days were uneventful with a progressive improvement of the patient, he made a good neurological recovery, was extubated successfully and was eventually transferred to the cardiology ward where he remained up until the eventual discharge from the hospital at the sixteenth day after admission.

\section{Discussion}

The signs of intra-abdominal bleeding are non-specific and usu- 
ally the patient presents himself with abdominal distention and pain and a history of blunt trauma. If left untreated, an active bleeding will lead to the swift death of the patient due to large volume of blood that can be lost into the abdominal cavity. In this clinical case, the identification of clinical degradation on a pattern that did not fit the initial diagnosis of myocardial infarction was key, leading to the quick identification of a second problem. The most sensitive diagnostic tool for the diagnosis of abdominal bleeding is a computed tomographic angiography and it also permits the distinction between an active and an inactive bleeding [1]. Upon identification of the bleeding the management strategy needs to be decided, with more unstable patients with multiples injuries necessitating emergent abdominal exploration [2] and patients with more favourable haemodynamic profile and single lesions being better candidates for an endovascular approach [3]. In this clinical case an endovascular approach was chosen since there was only a single bleeding vessel and the haemorrhagic shock was successfully offset with vasopressors and blood transfusions.

Although it was not possible to identify the definite reason for the hepatic artery rupture, spontaneous hemoperitoneum has been described as a rare and serious complication following cardiopulmonary resuscitation [4], with major liver injury (rupture/laceration, haemorrhage/haematoma) occurring in $0.6 \%$ of patients following CPR [5]. The authors consider it as the most probable cause. Other possibilities include a spontaneous bleeding associated with the use of antiplatelet and anticoagulant medication or as a direct complication of the PCI procedure.

\section{Conclusion}

This case report illustrates the successful management with an endovascular mean of an active bleeding in an unstable patient. The authors also hope that this case will help emphasize the need to always consider alternative problems when a patient deteriorates and that fixating on the initial diagnosis may be erroneous and lead to severe consequences.

\section{Bibliography}

1. Iacobellis F., et al. "Dual-phase CT for the assessment of acute vascular injuries in high-energy blunt trauma: the imaging findings and management implications". British Journal of Radiology 89.1061 (2016): 20150952.

2. Weber DG., et al. "Damage control surgery for abdominal emergencies". British Journal of Surgery 101.1 (2014): 109-118.
3. Chan YC., et al. "Management of spontaneous and iatrogenic retroperitoneal haemorrhage : conservative management, endovascular intervention or open surgery?". International Journal of Clinical Practice 62.10 (2008): 1604-1613.

4. Næss PA., et al. "Minimal invasive treatment of life- threatening bleeding caused by cardiopulmonary resuscitation-associated liver injury : a case report". Journal of Medical Case Reports (2016): 1-4.

5. Meron G., et al. "Cardiopulmonary resuscitation-associated major liver injury". Resuscitation 75.3 (2007): 445-453.

\section{Volume 5 Issue 6 June 2021}

(C) All rights are reserved by José João Martins., et al.. 